\title{
CONTROL OF OPTICAL VORTEX DISLOCATIONS USING OPTICAL METHODS
}

\author{
P. Stanislovaitis and V. Smilgevičius \\ Laser Research Center, Vilnius University, LT-10222 Vilnius, Lithuania \\ E-mail: voveraitis@gmail.com
}

Received 22 March 2012; revised 23 May 2012; accepted 20 September 2012

\begin{abstract}
In this paper we present the results of theoretical and experimental investigations of the optical vortex screw-dislocation position control based on optical vortex interference with the Gaussian beam. Optical vortices can be controlled by joining a Gaussian beam with a collinear optical vortex beam and changing the Gaussian beam intensity and phase. It is shown theoretically and experimentally that in this way it is possible to precisely change the optical vortex screw-dislocation position in plane transverse to propagation direction.
\end{abstract}

Keywords: optical vortices, interference, Laguerre-Gaussian beams

PACS: 42.25.Dd, 42.60.Jf

\section{Introduction}

In 1974 Nye and Berry [1] showed that wavefronts can contain screw and edge dislocations. Since then wavefront dislocations have become a subject of intense scientific investigation. For practical applications of optical vortices in optical tweezers, scanning microscopy, laser micromachining, it is necessary to control its position in plane transverse to propagation direction. There are a few methods often used for controlling the position of optical vortices: a computer controlled transparent liquid-crystal matrix, a moveable vortex lens and control of optical vortex dislocations using its interference with the Gaussian beam. All methods referred to above have advantages and disadvantages. So, the use of a transparent liquidcrystal matrix for optical vortex position control [2] is a very simple practical method, but it is limited by the optical damage threshold $(\sim 100 \mathrm{~kW} /$ $\mathrm{cm}^{2}$ ) of a transparent liquid-crystal matrix. The moveable vortex lens method [3] requires stable and precise mechanical mountings but does not have limitation on the optical beam damage. The control of the position of optical vortex dislocations by using its interference with the Gaussian beam is an alternative method. In the presence of the coherent background beam dark cores of the optical vortices do not disappear, but change their positions [ [4]. This makes it possible to control them by using another beam. Such method of control in a nonlinear medium was proposed in [5]. The interference of two collinear LaguerreGaussian beams of zero radial indices was investigated in [6, 7], and vortex propagation dynamics due to the Gouy phase was analysed in [8].

In this paper we present the results of theoretical and experimental investigations of the optical vortex screw-dislocation position control based on the optical vortex interference with the Gaussian beam. The optical vortex screw-dislocation position is controlled by joining a Gaussian beam with a collinear optical vortex beam and changing the Gaussian beam intensity and phase. It is shown theoretically and experimentally that in this way it is possible to precisely change the optical vortex screw-dislocation position in plane transverse to propagation direction. 


\section{Theoretical background}

As initial conditions we have chosen a LaguerreGaussian optical vortex of topological charge $m=1$ co-propagating with a collinear Gaussian beam, and their spot sizes and wavefront curvature radii coincide. Their amplitudes on line $z=$ const and $y=0$ are given in Fig. 1. Both beams are assumed to be polarised linearly and in the same direction. We can find optical vortex dislocation positions by their characteristic property that the intensity of light in these places is equal to zero. Zero intensity does not necessarily indicate an optical vortex (e. g. radial polarisation beams [9]), but in this paper we talk about linearly polarised Laguerre-Gaussian beams. As seen from Fig. 1 (this picture shows amplitudes of an optical vortex and Gaussian beams), for $x<0$ interference will be destructive. There is a unique point, where due to destructive interference the waves will cancel out each other (as seen in Fig. 1). The dislocation will exist at this point. The position of this dislocation will depend on the intensity ratio of the beams, since if we increase the Gaussian beam's amplitude (Fig. 11) while the amplitude of the optical vortex stays the same, we will see that the zero-intensity point moves from the centre to the edge of the beam (also see [4]). This allows controlling the distance of dislocation from the beam centre by changing the intensity ratio of the beams. Also, if the phase difference between

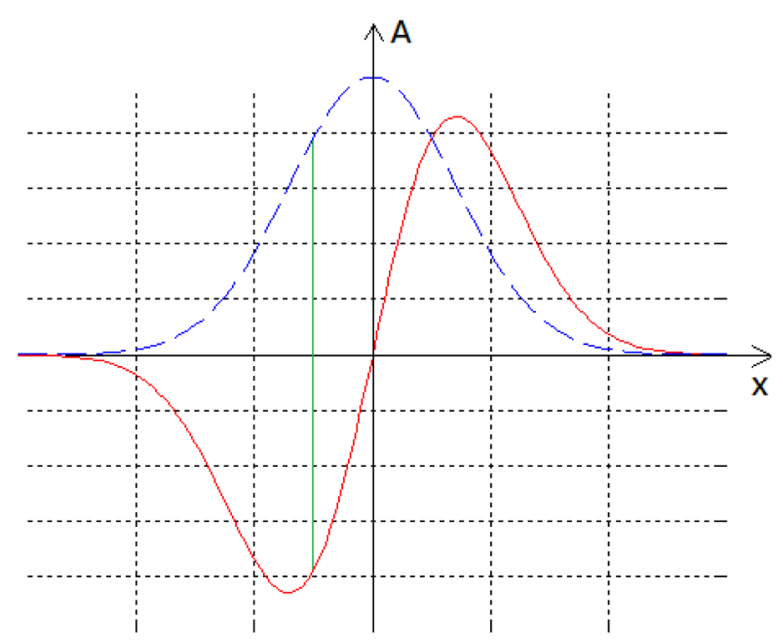

Fig. 1. Complex amplitudes of the LG beam with topological charge $m=1$ (solid, red online) and Gaussian beam (dashed, blue online) on line $y=z=0$. these beams changes, the whole "combined beam" will rotate around its axis, thus allowing us to control the angular position of the dislocation [6].

Consider two collinear Laguerre-Gaussian beams of topological charges $m$ and $l$. Their complex amplitudes are given by Eqs. (1a) and (1b):

$$
\begin{aligned}
& E_{1}(\rho, \varphi, z)=A_{01} \frac{W_{01}}{W_{1}}\left(\frac{\rho}{W_{1}}\right)^{|m|} \\
& e^{-\frac{\rho^{2}}{W_{1}^{2}} e^{i\left(k z+m \varphi+k \frac{\rho^{2}}{2 R_{1}}-\eta_{1}\right)},} \\
& E_{2}(\rho, \varphi, z)=A_{02} \frac{W_{02}}{W_{2}}\left(\frac{\rho}{W_{2}}\right)^{|l|} \\
& e^{-\frac{\rho^{2}}{W_{2}^{2}} e^{i\left(k z+l \varphi+k \frac{\rho^{2}}{2 R_{2}}-\eta_{2}\right)},}
\end{aligned}
$$

where $W_{01}$ and $W_{02}$ are waist radii of the beams, $W_{1}(z)$ and $W_{2}(z)$ are spot radii, $R_{1}(z)$ and $R_{2}(z)$ are radii of wavefront curvature, and $\eta_{1}(z)$ and $\eta_{2}(z)$ are Gouy phases.

In these equations we accept that $W_{01}=W_{02}=W_{0}$, $W_{1}(z)=W_{2}(z)=W(z)$, and $R_{1}(z)=R_{2}(z)=R(z)$, where $W(z)=W_{0}\left[1+\left(z / l_{\mathrm{d}}\right)^{2}\right]^{1 / 2}, R(z)=z\left[1+\left(l_{\mathrm{d}} / z\right)^{2}\right)$, and $l_{\mathrm{d}}=k W_{0}^{2} / 2$. Also, we can accept that $|m|>|l|$ and $m>l$. Dislocations will exist at the points where the sum of these beam amplitudes will be zero:

$$
E=E_{1}+E_{2}=0
$$

This means that both real and imaginary parts of $E$ must be ual to zero:

$$
\begin{aligned}
& \operatorname{Re}(E)=0, \\
& \operatorname{Im}(E)=0,
\end{aligned}
$$

From Eqs. (3a) and (3b) we can find positions of vortex dislocations in cylindrical coordinates in plane $z=$ const.

$$
\begin{aligned}
& \rho=W\left(\frac{A_{2}}{A_{1}}\right)^{\frac{1}{|m|-|l|}}, \\
& \varphi=\frac{(2 n+1) \pi+\Phi_{0}}{m-l},
\end{aligned}
$$




$$
\begin{aligned}
& A_{1}=A_{01} \frac{W_{0}}{W(z)}, \\
& A_{2}=A_{02} \frac{W_{0}}{W(z)},
\end{aligned}
$$

where $\Phi_{0}$ is the arbitrary phase difference between the beams plus the Gouy phase difference, and $n$ is an integer $(0 \leq n<|m-l|$ for each dislocation at $\rho \neq 0$ ). Gouy phases depend only on $z$ coordinate and in plane $z=$ const they are constant; therefore, we put them into constant $\Phi_{0}$. Also, if $l \neq 0$, a dislocation of topological charge $m-l$ exists at $\rho=0$.

Equations (4a) and (4b) show that the radial position of optical vortices depends only on the ratio of amplitudes and the angular position depends only on the phase difference. However, this conclusion is only valid if the beam wavefront curvature radii coincide. Otherwise, the angular position of vortex dislocations will also depend on the amplitude ratio.

Equation (4a can be rewritten in terms of maximum beam intensities (6):

$$
\rho=\frac{W}{\sqrt{2 e}} \sqrt{\frac{I_{2 \max }}{I_{1 \max }} \frac{|m|^{|m|}}{|l|^{|l|}}} \frac{1}{|m|-|l|} .
$$

Numerical simulations for various intensity ratios and phase differences for the case $m=1$ and $l=0$ are shown in Fig. 2. As the intensity of the
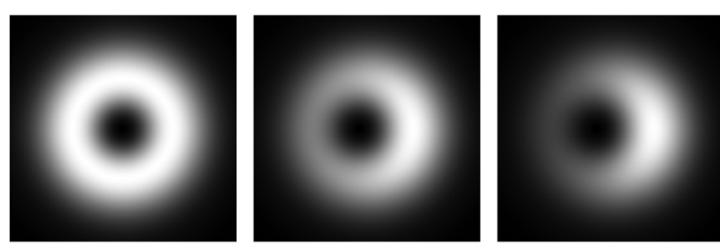

$$
I_{2 \max }=0 \quad I_{2 \max }=0.0543 I_{1 \max } I_{2 \max }=0.2175 I_{1 \max }
$$

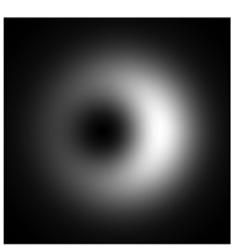

$\Phi_{0}=0^{\circ}$

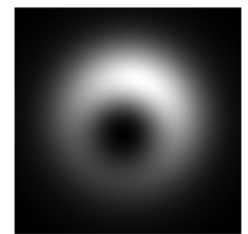

$\Phi_{0}=72^{\circ}$

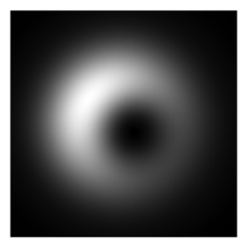

$\Phi_{0}=144^{\circ}$
Fig. 2. Intensity distributions of the combined beam consisting of a Gaussian beam and vortex of topological charge $m=1$ (numerical simulation). Top row shows intensity distributions with different Gaussian beam intensities, bottom row shows intensity distributions with various phase differences between the beams. background beam $(l=0)$ increases, the dislocation moves from the centre to the edge of the beam. When the phase difference between the beams is changed, the whole combined beam rotates around its central axis.

\section{Creation and annihilation of secondary dislocations}

If diameters of the beams do not coincide, there is a possibility that secondary dislocations will appear. Also, under certain conditions secondary dislocations will annihilate with primary dislocations and the resulting beam will not contain any dislocations (except for dislocation at $\rho=0$ if it exists). Theoretical analysis has shown that secondary dislocations exist under the following conditions:

$$
\sigma<1
$$

$$
\begin{aligned}
& \sigma>1 ; q<\left(\frac{|m|-|l|}{2 e}\right)^{\frac{|m|-|l|}{2}} \frac{\sigma^{|m|}}{\left(\sigma^{2}-1\right)^{\frac{|m|-|l|}{2}}}, \\
& \sigma>1 ; q>\left(\frac{|m|-|l|}{2 e}\right)^{\frac{|m|-|l|}{2}} \frac{\sigma^{|m|}}{\left(\sigma^{2}-1\right)^{\frac{|m|-|l|}{2}}},
\end{aligned}
$$

where $\sigma=W_{2} / W_{1}, q=A_{2} / A_{1}$, and $A_{1}, A_{2}$ are given by Eq. (5). When $\sigma$ satisfies the condition under Eq. (7a) (the beam with a higher topological charge has a larger radius than the beam with a lower topological charge), secondary dislocations do not exist. When $q$ and $\sigma$ satisfy conditions under Eq. (7b), secondary dislocations exist. As the intensity of the beam with a lower topological charge increases, dislocations get closer to each other and finally annihilate. When conditions under Eq. (7c) are satisfied, secondary dislocations annihilate with primary dislocations and the resulting beam does not contain any dislocations (except for dislocation at $\rho=0$ if it exists).

Conditions under Eqs. $(7 \mathrm{a}-\mathrm{c})$ can be shown graphically. Their graphical representations for different topological charges are given in Figs. 3-5. In the area marked " 1 ", secondary dislocations do not exist. In the area marked "2", secondary dislocations exist. As the intensity of the beams with a lower topological charge increases, the dislocations get 

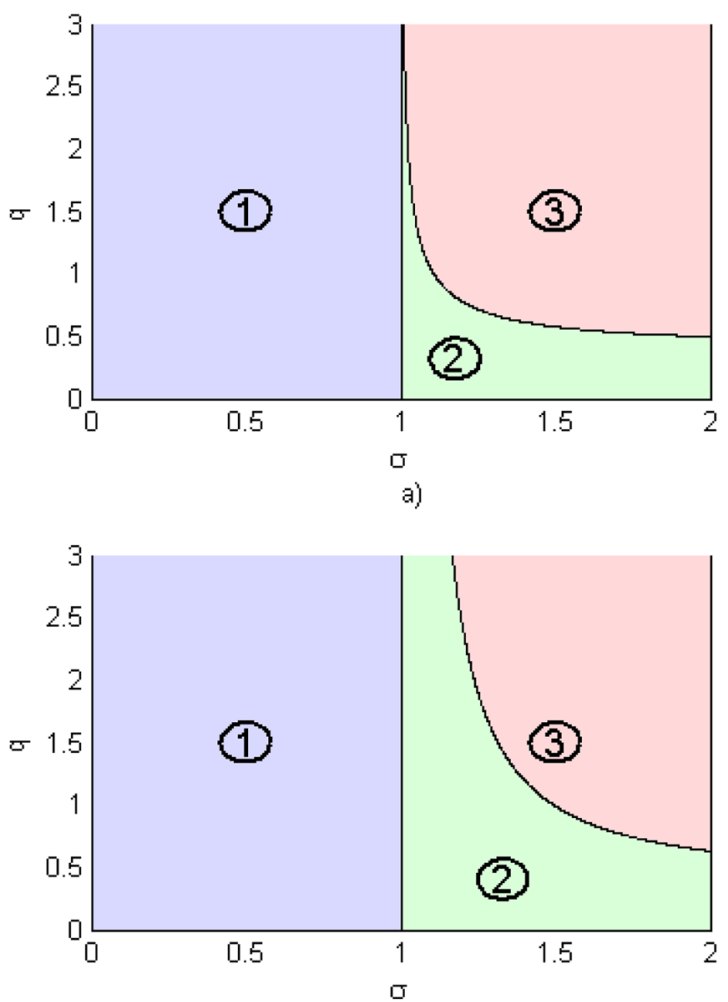

b)

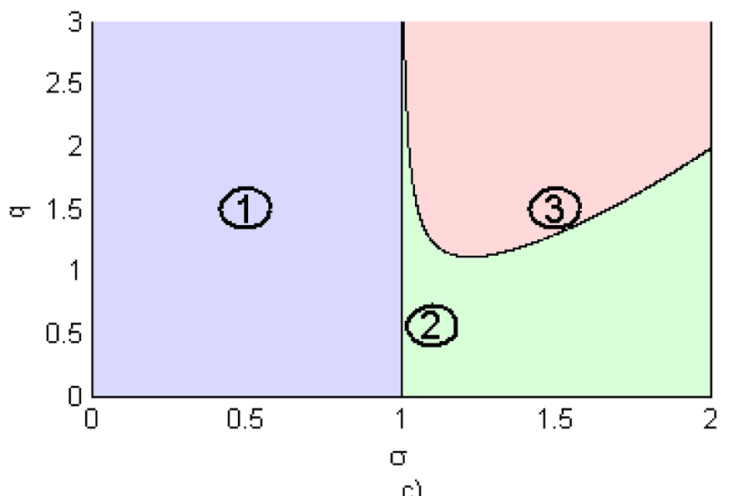

c)

Fig. 3. Graphical representation of Eqs. $(7 \mathrm{a}-\mathrm{c})$ for various topological charges: (a) $m=1, l=0$, (b) $m=3, l=0$, (c) $m=3, l=2$. Area 1 represents Eq. (7a), area 2 represents Eq. (7b), area 3 represents Eq. (7c).

closer to each other and when the combined beam parameters enter the area marked " 3 ", secondary dislocations annihilate and the resulting beam does not contain any dislocations (except for the dislocation in the centre if it exists $(l \neq 0))$.

\section{Experiment}

A computerised vortex controller device was implemented experimentally. The experimental set- up is shown in Fig. 4. A Gaussian beam from the HeNe laser was split into two beams with the beam splitter $B S 1$. One part of the beam was then sent through the vortex hologram $H$, forming a single topological charge optical vortex. Another part of the beam reflected from mirror $M 2$ and went through lens $L 2$, filter $F$, and lens L3. Mirror M2 was mounted on the piezoelectric translation stage and thus could change the Gaussian beam phase by moving mirror $M 2$ with $\lambda / 200$ precision. For intensity control a variable density ND filter disk was mounted on the stepper motor axis, which was controlled from the computer. This allowed changing the intensity of the Gaussian beam. The telescope consisting of $L 2$ and $L 3$ was used to control the wavefront radius of the Gaussian beam. Both mirror $M 2$ and filter $F$ were controlled from the computer. Both beams were then recombined at $B S 2$ and sent to the CCD camera. Intensity distributions with various phase differences between the vortex and Gaussian beam registered with the CCD camera are shown in Fig. 5. The experiment was carried out with vortices of topological charges $m=1$ and 2 .

Finally, we theoretically and experimentally showed that it is possible to move vortex dislocations from the centre to the edges of the beam when the background beam intensity increases and rotate the combined beam linearly by increasing phase difference. A vortex with the topological charge $m=2$, splitting dislocation into two single topological charge dislocations, was observed. This was confirmed using the interference of a Gaussian beam and the combined beam. In the interference pattern, two forked interference fringes were seen, meaning that there were two dislocations in the combined beam. Also, for the higher topological charge vortices a slower rotation of the beam due to the phase difference took place. This can be seen from Eq. (4b), as the denominator was equal to $m-l$. If the background beam is Gaussian, then $l=0$, meaning that angular coordinates of the dislocations are inversely proportional to the topological charge $m$ of the vortex beam.

\section{Conclusions}

In conclusion, we have theoretically and experimentally shown an effective method to control optical vortex dislocation positions by using a collinear coherent background beam. In our work we 


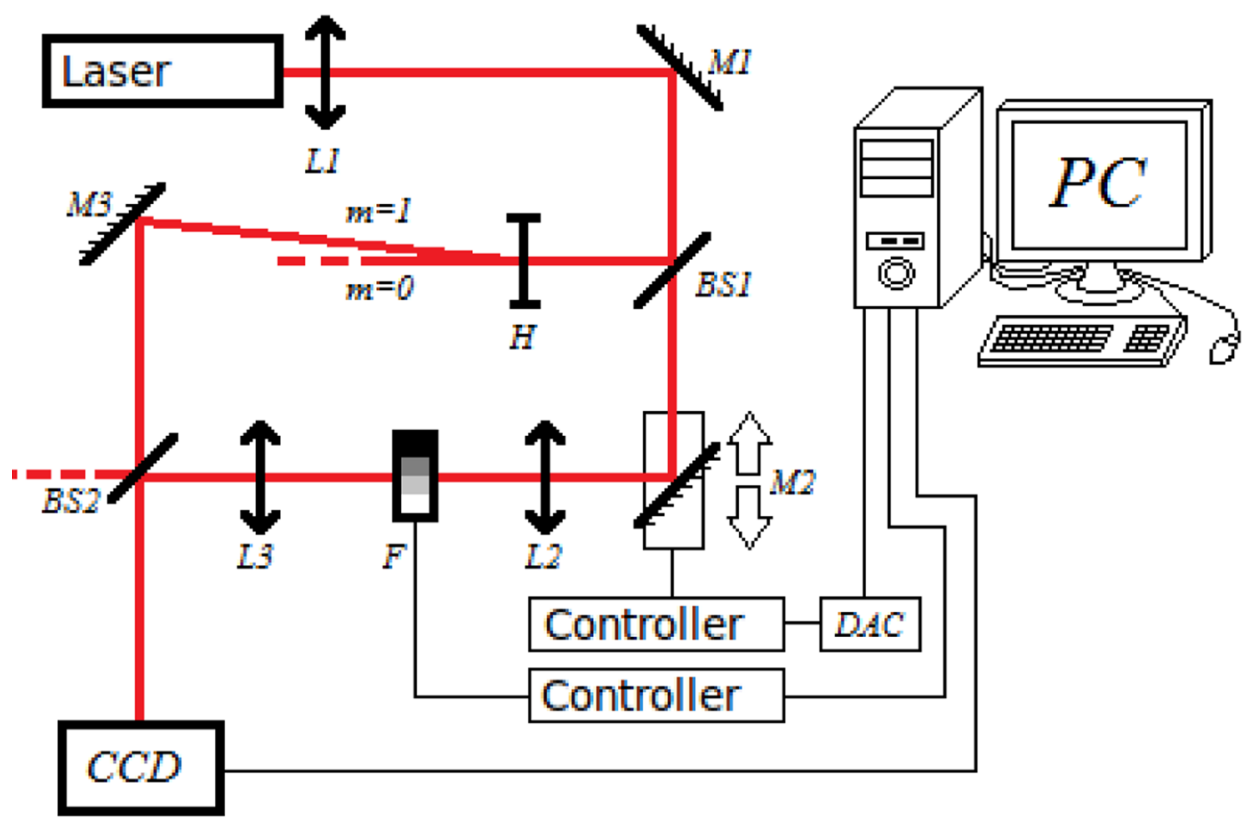

Fig. 4. Experimental set-up: M1...M3 mirrors, L1...L3 lenses, PC computer, DAC digital-to-analog converter, $B S 1$ and $B S 2$ beam splitters, $H$ optical vortex hologram, $F$ variable density ND filter disk.
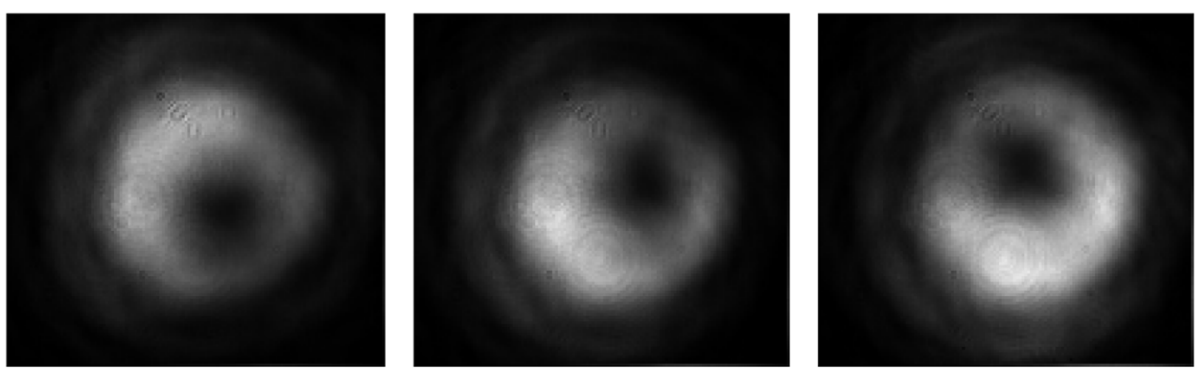

a)
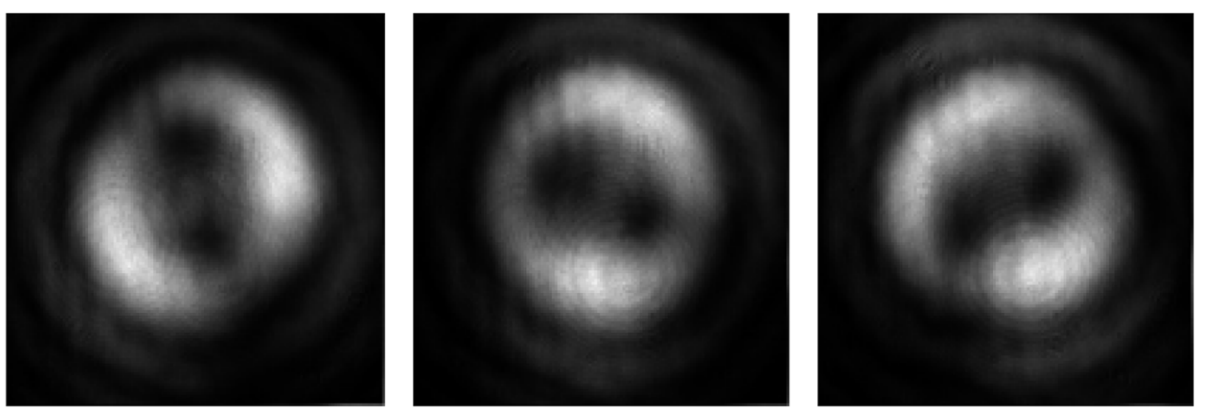

b)

Fig. 5. Intensity distributions of the combined beam with various phase differences between beams (experimental results with vortices of topological charge 1 (a) and 2 (b)). Phase difference between the beams changes from left to right: $\Delta \Phi=0^{\circ}, \Delta \Phi=90^{\circ}$, $\Delta \Phi=180^{\circ}$. 
have derived formulas for calculating dislocation positions for combined Laguerre-Gaussian beams of a zero radial index. The advantage of this method is that it is not limited by the optical damage threshold. However, dislocations of higher than zero topological charge split into unit topological charge dislocations and also the dislocation range of motion is limited. Our investigations show that the proposed method could be extended to control properties of vortex assemblies.

\section{Acknowledgements}

This work was supported by the INTEGRA project of the Lithuanian Agency for Science, Innovation and Technology and partly by LaserLab Europe under HAPPIE JRA.

\section{References}

[1] J.F. Nye and M.V. Berry, Dislocations in wave trains, Proc. R. Soc. London A 336, 165-190 (1974).

[2] V. Bingelyte, J. Leach, J. Courtial, and M.J. Padgett, Optically controlled three-dimensional rotation of microscopic objects, Appl. Phys. Lett. 82, 020307 (2003).

[3] J. Masajada, M. Leniec, and I. Augustyniak, Optical vortex scanning inside the Gaussian beam, J. Opt. 13, 035714 (2011).

[4] M.S. Soskin, V.N. Gorshkov, M.V. Vasnetsov, J.T. Malos, and N.R. Heckenberg, Topological charge and angular momentum of light beams carrying optical vortices, Phys. Rev. A 56(5), 4064 (1997).

[5] J. Christou, V. Tikhonenko, Y.S. Kivshar, and B. Luther-Davies, Vortex soliton motion and steering, Opt. Lett. 21(20), 1649 (1996).

[6] E.J. Galvez, N. Smiley, and N. Fernandes, Composite optical vortices formed by collinear Laguerre-Gaussian beams, Proc. SPIE 6131, 19-26 (2006).

[7] G. Molina-Terriza, J. Recolons, and L. Torner, The curious arithmetic of optical vortices, Opt. Lett. 25(16), 1135 (2000).

[8] S.M. Baumann, D.M. Kalb, L.H. MacMillan, and E.J. Galvez, Propagation dynamics of optical vortices due to Gouy phase, Opt. Express 17(12), 9818 (2009).

[9] A.V. Nesterov, V.G. Niziev, and V.P. Yakunin, Generation of high power radially polarized beam, J. Appl. Phys. D 32, 2871-2875 (1999).

\title{
ŠVIESOS SŪKURIŲ DISLOKACIJŲ VALDYMAS OPTINIAIS METODAIS
}

\author{
P. Stanislovaitis, V. Smilgevičius \\ Vilniaus universiteto Lazeriu tyrimo centras, Vilnius, Lietuva
}

\section{Santrauka}

Straipsnyje pristatomi šviesos sūkurių dislokacijų valdymo (naudojant interferenciją su Gauso pluoštu) teorinių ir ekperimentinių tyrimų rezultatai. Šviesos sūkuriai gali būti valdomi suvedant Gauso pluoštą su koli- neariu šviesos sūkurio pluoštu ir keičiant Gauso pluošto fazę bei intensyvumą. Ekperimentiškai ir teoriškai parodyta, kad tokiu būdu i̇manoma tiksliai keisti šviesos sūkurio dislokacijų pozicijas plokštumoje, statmenoje pluošto sklidimo krypčiai. 